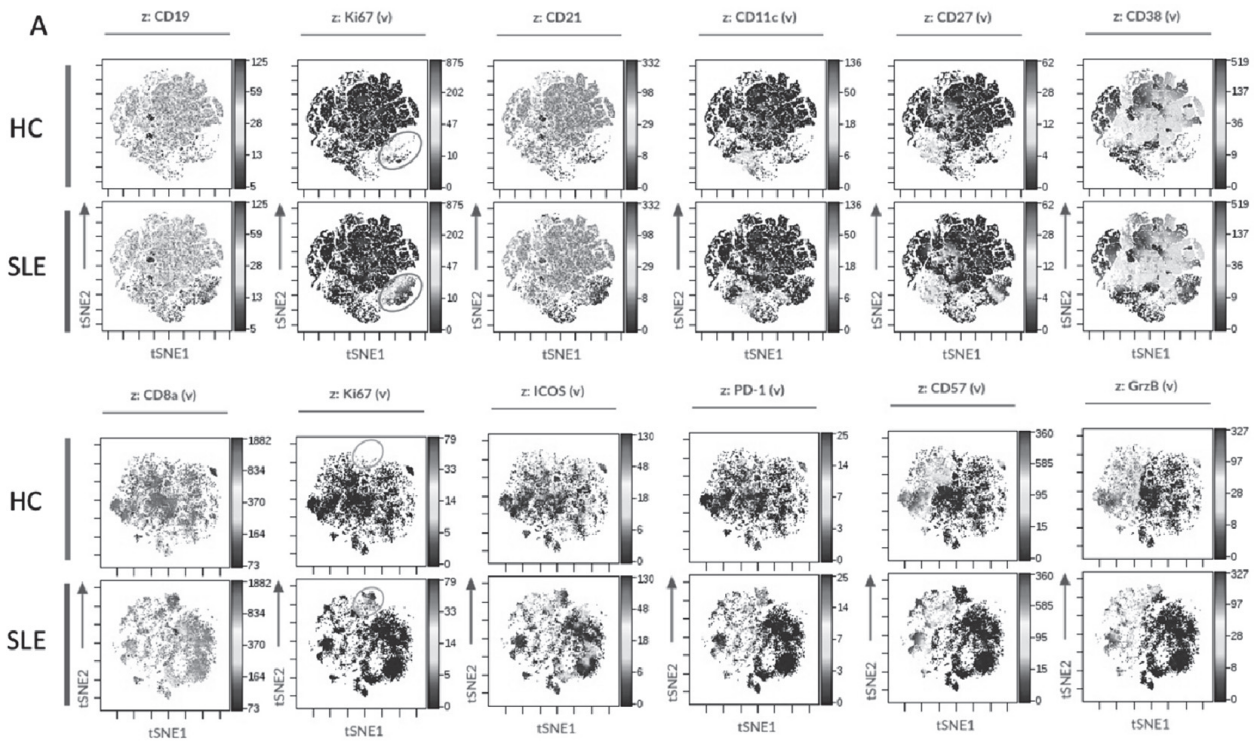

B

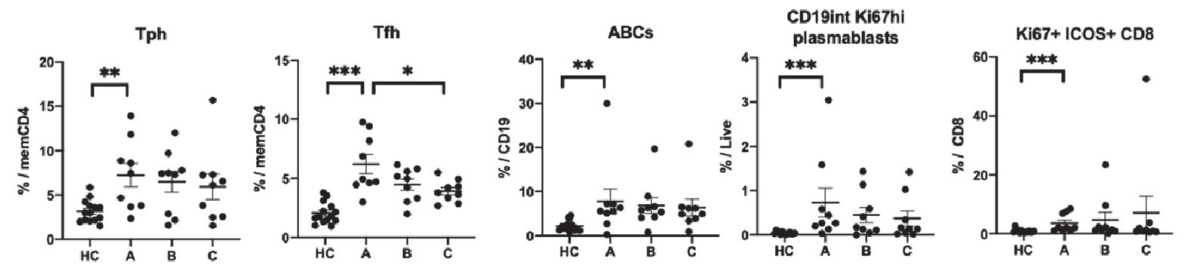

C

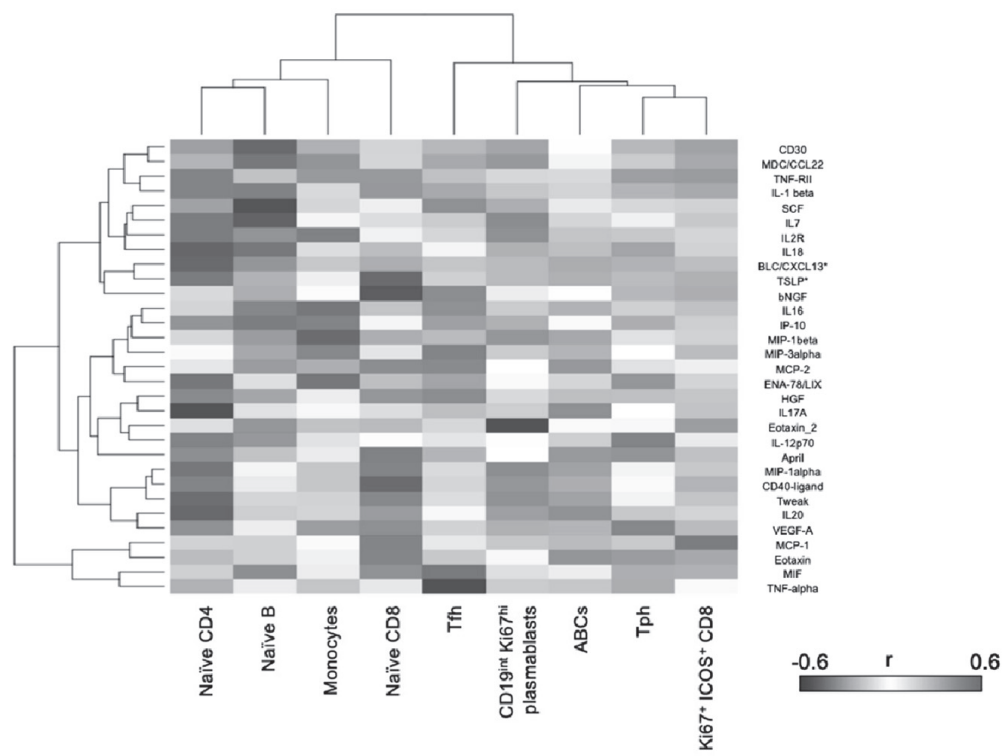

Abstract 602 Figure 1 Longitudinal CyTOF and cytokine analyses of newly diagnosed SLE. (A) Expanded two Ki67+ populations in PBMCs of SLE patients. (B) Longitudinal CyTOF analysis of PBMCs in SLE patients. (C) A hierarchical clustering heatmap with immune cell frequencies and cytokines in SLE.

and CD19 ${ }^{\text {int }}$ Ki67 ${ }^{\text {hi }}$ plasmablasts. These associated populations, but not Tfh cells, were also significantly correlated with CXCL13 and TSLP (figure 1C).

Conclusions This longitudinal immunophenotyping and cytokine profiling approach highlights persistent activation of a Tph-CXCL13-ABC-plasmablasts axis in both early and established phases of SLE.

Acknowledgments We thank the patients who donated samples and medical staffs at the Hospital.

\section{AUTOREACTIVITY DRIVES INCREASED METABOLISM IN T CELLS FROM SLE PATIENTS?}

${ }^{1}$ Kriti Bahl, ${ }^{1,2}$ Darienne Myers, 'Jeroen Roose*. ${ }^{1}$ University of California, San Francisco (UCSF), CA, USA; ${ }^{2}$ Rheos Medicines

10.1136/lupus-2021-lupus21century.35

Background Auto-reactive CD4+ T cells play an important role in the pathogenesis of SLE and RA. Self-reactive CD4+ 
$\mathrm{T}$ cells in their abnormal interface with $\mathrm{B}$ cells cause tissue damage, and auto-antigen release, leading to further activation and differentiation of self-reactive CD4+ T cells. Recognition of $\mathrm{MHC} /$ Self-peptide plays a causative role in autoimmunity 1,2. How this leads to abnormal activation of $\mathrm{CD} 4+\mathrm{T}$ cells is not clear.

Methods We optimized a,single cell resolution method - barcoded phosphoflow 3- to quantitatively measure intracellular signaling pathways in $\mathrm{T}$ cells that encounter MHC/Self-peptide. We unleashed this method on $\mathrm{T}$ cells from various genetic mouse models and combined this with a novel methodology to measure cell metabolism, SCENITH, also with single cell resolution.

Results RasGRP1 has been associated with autoimmune diseases, but the genetic basis or impact on $\mathrm{T}$ cells is not known. Likewise, mTORC1 signals and altered cell metabolism links to autoimmunity and the mTOR inhibitor Rapamycin reduces disease severity in autoimmune patients and mouse models. In our studies, we uncovered a novel Rasgrp1-mTORC1 pathway that is selectively triggered when $\mathrm{T}$ cells see MHC/Self-peptide (see figure 1). In our Rasgrp1Anaef mouse model 4,5 a single R519G point mutation results in aberrantly elevated Rasgrp1Anaef-mTORC1 signals that drive resting $\mathrm{T}$ cells out of their naïve state, leading to autoimmune pathology 4. Mechanistically, this elevated Rasgrp1Anaef-mTORC1 signal does not lead to altered gene expression, but instead, results in unwanted translation of mRNA targets in naïve T cells 4 .

Conclusions Thus, Rasgrp1-mTORC1 signals are selectively triggered when $\mathrm{T}$ cells see self and are highest when $\mathrm{T}$ cells see self the strongest (auto-reactive) 4. We are investigating the fundamental properties of Rasgrp1-mTORC1 signals and effects on metabolism and protein translation. We are complementing these directions with efforts on SLE and RA patient samples and desire to expand this area through Dr. Roose's roles in UCSF ImmunoX and AutoImmunoProfiler.
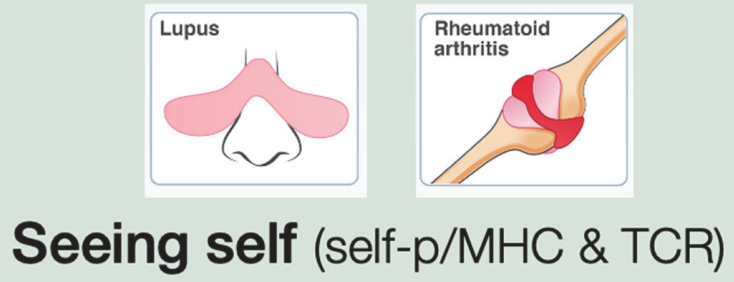

TONIC SIGNAL

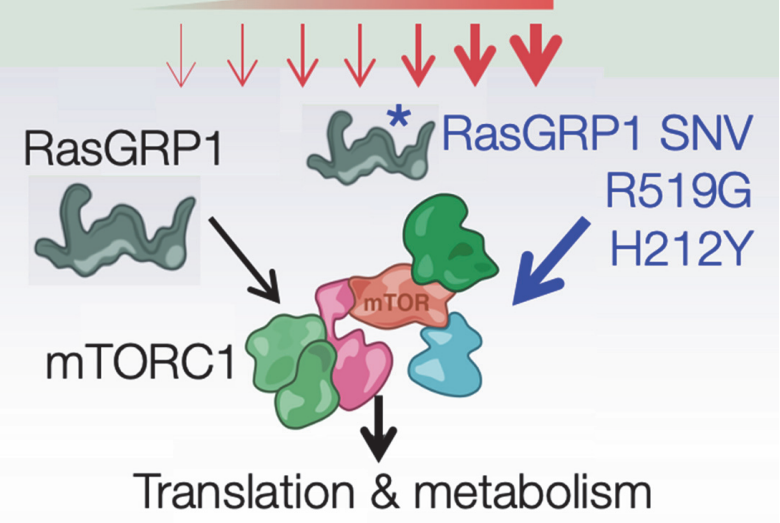

Abstract 603 Figure 1

\section{REFERENCES}

1. Myers DR, et al. Immunol Rev. 2019;291.

2. Myers DR, et al. Trends Immunol. 2017:38.

3. Kulhanek KR, et al. STAR Protoc. 2020;1.

4. Myers DR, et al. Cell Reports. 2019;27.

5. Daley SR, et al. elife. 2013;2.

Acknowledgments We thank all members of the Roose lab for helpful discussions and the UCSF Parnassus Flow Cytometry Core for assistance with cell sorting (NIH P30 DK063720), the UCSF Genomics CoLab for RNA-Seq library preparation, the UCSF Center for Advanced Technology for sequencing, Max Horlbeck for assistance with bioinformatics. These studies were supported by grants from the NSF-GRFP (1650113 to DRM), the Marie Curie IOF (\#PIOF-GA-2012-328666), and the NIH-NIAID (R01-AI104789 and P01-AI091580 to JPR).

\section{0 - Macrophage in SLE}

\section{THERAPY OF DIFFUSE ALVEOLAR HEMORRHAGE IN EXPERIMENTAL LUPUS WITH RECOMBINANT MYXOMAVIRUS PROTEIN SERP-1}

${ }^{1}$ Haoyang Zhuang, ' $S$ huhong Han, ${ }^{2} \mathrm{Li}$ Lu, ${ }^{1,2}$ Westley H Reeves*. 'Division of Rheumatology, Allergy and Clinical Immunology; ${ }^{2}$ Department of Pathology, Immunology and Laboratory Medicine, University of Florida, Gainesville, FL USA

\subsection{6/lupus-2021-lupus21century.36}

Background Although diffuse alveolar hemorrhage (DAH) is an uncommon complication of SLE, over half of patients die. B6 mice with pristane-induced lupus and $\mathrm{DAH}$ are an animal model of this disorder. ANCA-negative small vessel vasculitis with hemorrhage and hemosiderin-laden macrophages are seen in lung tissue from both SLE patients and mice with DAH. DAH in pristane-lupus is prevented by depleting macrophages whereas neutrophil depletion has no effect. IL-10 deficiency exacerbates DAH while macrophage repolarization induced by liver $\mathrm{X}$ receptors (LXR) prevents it. The myxomavirusencoded serpin Serp-1 impairs macrophage activation and plasminogen activation and blocks DAH caused by MHV68 infection. We asked whether it also could block DAH in pristaneinduced lupus.

Methods B6 mice were treated with pristane \pm daily injection of recombinant Serp-1. Severity of DAH was assessed at day14 by gross pathology, H\&E, and Prussian blue staining. LXR activation was assessed by measuring Nr1h3 mRNA (encoding LXR $\alpha$ ) and flow cytometry for the LXRregulated ATP Binding Cassette Subfamily A Member 1 (ABCA1) protein. The effect of Serp-1 on macrophage polarization was investigated in lung tissue and in RAW264.7 cells.

Results DAH was prevented by recombinant Serp-1. Serp-1 treatment repolarized macrophages toward an anti-inflammatory M2-like phenotype, increased expression of the transcription factor.

Kruppel-like factor-4 (Klf4), which regulates IL-10 production, and increased expression of $\mathrm{Nr} 1 \mathrm{~h} 3$, which along with Klf4 regulates M2 polarization. Serp-1 also corrected a lupusassociated deficit of LXR-regulated reverse cholesterol transporter protein ABCA1. In RAW264.7 cells, Serp-1 increased Klf4 mRNA levels and LPS-stimulated IL-10 secretion while reducing TNF $\alpha$. Although Serp-1 affects both thrombotic and 